\title{
Vitamina D e doenças endocrinometabólicas
}

\author{
Vitamin D and endocrine diseases
}

Natielen Jacques Schuch ${ }^{1}$, Vivian Cristina Garcia' ${ }^{1}$, Ligia Araújo Martini ${ }^{1}$

\section{RESUMO}

Atualmente, a insuficiência/deficiência de vitamina $D$ tem sido considerada um problema de saúde pública no mundo todo, em razão de suas implicações no desenvolvimento de diversas doenças, entre elas, o diabetes melito tipo 2 (DMT2), a obesidade e a hipertensão arterial. A deficiência de vitamina $\mathrm{D}$ pode predispor à intolerância à glicose, a alterações na secreção de insulina e, assim, ao desenvolvimento do DMT2. Esse possível mecanismo ocorre em razão da presença do receptor de vitamina $D$ em diversas células e tecidos, incluindo células- $\beta$ do pâncreas, no adipócito e no tecido muscular. Em indivíduos obesos, as alterações do sistema endócrino da vitamina $D$, caracterizada por elevados níveis de $P T H$ e da $1,25(\mathrm{OH})_{2} \mathrm{D}_{3}$ são responsáveis pelo feedback negativo da síntese hepática de $25-\mathrm{OHD}_{3}$ e também pelo maior influxo de cálcio para o meio intracelular, que pode prejudicar a secreção e a sensibilidade à insulina. Na hipertensão, a vitamina $D$ pode atuar via sistema renina-angiotensina e também na função vascular. Há evidências de que a $1,25(\mathrm{OH})_{2} \mathrm{D}_{3}$ inibe a expressão da renina e bloqueia a proliferação da célula vascular muscular lisa. Entretanto, estudos prospectivos e de intervenção em humanos que comprovem a efetividade da adequação do status da vitamina D sob o aspecto "prevenção e tratamento de doenças endocrinometabólicas" são ainda escassos. Mais pesquisas são necessárias para se garantir o benefício máximo da vitamina D nessas situações. Arq Bras Endocrinol Metab. 2009;53(5):625-33.

Descritores

Vitamina D; diabetes melito; obesidade; hipertensão

\begin{abstract}
Vitamin D insufficiency/deficiency has been worldwide reported in all age groups in recent years. It has been considered a Public Health matter since decreased levels of vitamin $D$ has been related to several chronic diseases, as type 2 diabetes mellitus (T2DM), obesity and hypertension. Glucose intolerance and insulin secretion has been observed during vitamin D deficiency, both in animals and humans resulting in T2DM. The supposed mechanism underlying these findings is presence of vitamin $D$ receptor in several tissues and cells, including pancreatic $\beta$-cells, adipocyte and muscle cells. In obese individuals, the impaired vitamin D endocrine system, characterized by high levels of PTH and $1,25(\mathrm{OH})_{2} \mathrm{D}_{3}$ could induce a negative feedback for the hepatic synthesis of $25(\mathrm{OH}) \mathrm{D}$ and also contribute to a higher intracellular calcium, which in turn secrete less insulin and deteriorate insulin sensitivy. In hypertension, vitamin $D$ could act on renin-angiotensin system and also in vascular function. Administration of $1,25(\mathrm{OH})_{2} \mathrm{D}_{3}$ could decreases renin gene expression and inhibit vascular smooth muscle cell proliferation. However, prospective and intervention human studies that clearly demonstrates the benefits of vitamin $D$ status adequacy in the prevention and treatment of endocrine metabolic diseases are lacking. Further research still necessary to assure the maximum benefit of vitamin $D$ in such situations. Arq Bras Endocrinol Metab. 2009;53(5):625-33.
\end{abstract}

Keywords

Vitamin D; diabetes mellitus; obesity; hypertension

Correspondência para: Ligia Araújo Martini Departamento de Nutrição, Faculdade de saúde pública da Universidade de São Paulo Av. Doutor Arnaldo, 715, 2andar 01246-904 - São Paulo, SP, Brasil Imartini@usp.br

Recebido em 14/Abr/2009 Aceito em 26/Abr/2009 


\section{VITAMINA D, NOVOS HORIZONTES}

A atuação da vitamina $\mathrm{D}$ em processos metabólicos é Lpesquisada desde o século 17 e foi objeto de prêmio Nobel em 1938 (1). Atualmente, são conhecidos aproximadamente 41 metabólitos da vitamina D e um hormônio principal, a $1,25(\mathrm{OH})_{2} \mathrm{D}_{3}$, que atua como ligante para o fator de transcrição nuclear VDR (do inglês vitamin $\mathrm{D}$ receptor, receptor da vitamina $\mathrm{D})$, regulando a transcrição gênica e a função celular em diversos tecidos. Há evidências de que 3\% do genoma humano seja regulado pela $1,25(\mathrm{OH})_{2} \mathrm{D}_{3}(1)$.

A vitamina $\mathrm{D}$ é bastante conhecida pela sua função no desenvolvimento e na manutenção do tecido ósseo, bem como pela manutenção da homeostase normal do cálcio e do fósforo. Porém, evidências recentes sugerem o envolvimento dessa vitamina em diversos processos celulares vitais, como: diferenciação e proliferação celular, secreção hormonal (por exemplo: insulina), assim como no sistema imune e em diversas doenças crônicas não transmissíveis (1-5).

Na maioria dos indivíduos, a síntese cutânea é a principal fonte de vitamina $\mathrm{D}$, sendo o restante obtido pela alimentação e pelo uso de suplementos (2). Após a síntese cutânea, a vitamina $\mathrm{D}$ entra na circulação e é transportada para o fígado, unida à proteína ligante da vitamina $\mathrm{D}(\mathrm{DBP})$. No fígado, ocorre a primeira hidroxilação para a $25(\mathrm{OH}) \mathrm{D}$, que será secretada no plasma. Para se tornar ativa, a 25(OH)D é metabolizada pela enzima 25-hidroxivitamina D l $\alpha$-hidroxilase (CYP27Bl) nos rins, formando $1,25(\mathrm{OH})_{2} \mathrm{D}_{3}$. A produção desse metabólito é controlada principalmente pela concentração de paratormônio (PTH), cálcio e fósforo séricos.

Os efeitos biológicos da forma ativa da vitamina $D$ são mediados pelo VDR, presente nos principais sítios de ação da vitamina $\mathrm{D}$, como rim, glândulas paratireoides, intestino e osso. No núcleo das células-alvo, a $1,25(\mathrm{OH})_{2} \mathrm{D}_{3}$ se associa ao VDR. Esse complexo se liga ao receptor de ácido retinoico (RXR), formando heterodímeros que atuam nos elementos-resposta da vitamina D (VDRE), iniciando, assim, a cascata de interações moleculares que modulam a transcrição de genes específicos (3).

Apesar de o rim ser o principal sítio de hidroxilação para a forma ativa da vitamina $D$, a presença de CYP27Bl e do VDR foi identificada em outros tecidos, como próstata, mama, cólon, pâncreas e células do sistema imune. Acredita-se, porém, que a hidroxilação extrarrenal tenha efeito autócrino e parácrino local e não faça parte da circulação (6).
O nível individual do status da vitamina D é mensurado por meio dos níveis plasmáticos da $25(\mathrm{OH}) \mathrm{D}$. A forma biologicamente ativa da vitamina $\mathrm{D}, 1,25(\mathrm{OH})_{2} \mathrm{D}_{3}$, não é indicada para esse propósito devido a razões como: a) os níveis plasmáticos da $1,25(\mathrm{OH})_{2} \mathrm{D}_{3}$ são rigidamente mantidos em concentração normais; b) os níveis plasmáticos de $25(\mathrm{OH}) \mathrm{D}$ são aproximadamente cem vezes maiores do que os de $\left.1,25(\mathrm{OH})_{2} \mathrm{D}_{3} ; \mathrm{c}\right)$ a hidroxilação da $25(\mathrm{OH}) \mathrm{D}$ a $1,25(\mathrm{OH})_{2} \mathrm{D}_{3}$ ocorre em diversos tecidos, cobrindo as necessidades locais; e d) a meia-vida da $1,25(\mathrm{OH})_{2} \mathrm{D}_{3}$ é de aproximadamente seis horas, enquanto a da $25(\mathrm{OH}) \mathrm{D}$, de duas a três semanas (7).

Os níveis séricos tidos como adequados ou não ainda são muito discutidos na literatura. Hollis (8) considera que o nível ótimo de vitamina $\mathrm{D}$ seria aquele necessário para manter o PTH em níveis adequados, visto que a deficiência de vitamina $\mathrm{D}$ leva à diminuição do cálcio sérico, o qual, em consequência, estimula as glândulas paratireoides a liberar o PTH, a fim de elevar a reabsorção renal e óssea do cálcio (8). Neste sentido, vários estudos têm encontrado um platô de absorção de cálcio e níveis adequados de PTH, com níveis de $25(\mathrm{OH}) \mathrm{D}$ próximos a $50 \mathrm{nmol} / \mathrm{L}(9-11)$.

Revisando estudos sobre os pontos de corte da vitamina D, Grant e Holick, em 2005 (12), propuseram que a concentração abaixo de $50 \mathrm{nmol} / \mathrm{L}$ seja considerada como deficiência de vitamina D; as concentrações entre 50 e 80 $\mathrm{nmol} / \mathrm{L}$ seriam indicadoras de insuficiência. Os valores de $25(\mathrm{OH}) \mathrm{D}$ normalmente são expressos em nmol/L ou $\mathrm{ng} / \mathrm{mL}$ ( $1 \mathrm{ng} / \mathrm{mL}$ corresponde a $2,496 \mathrm{nmol} / \mathrm{L})$. Os pontos de corte estão ilustrados na tabela 1 .

\begin{tabular}{|c|c|c|}
\hline $25-\mathrm{OHD}_{3}(\mathrm{ng} / \mathrm{mL})$ & $25-\mathrm{OHD}_{3}(\mathrm{nmol} / \mathrm{L})$ & Indicador de saúde \\
\hline$<20$ & $>50$ & Deficiência \\
\hline $20-32$ & $50-80$ & Insuficiência \\
\hline $32-100$ & $80-250$ & Suficiência \\
\hline $54-90$ & $135-225$ & Normal em países ensolarados \\
\hline$>100$ & $>250$ & Excesso \\
\hline$>150$ & $>325$ & Intoxicação \\
\hline
\end{tabular}

Adaptado de Grant e Holick (12).

Atualmente, a insuficiência/deficiência de vitamina D tem sido considerada um problema de saúde pública no mundo todo, em razão de suas implicações no desenvolvimento de diversas doenças (3). Em 2008, a publicação referente ao $22^{\text {nd }}$ Marabou Symposium: the changing faces of vitamin $D$ relatou insuficiência de vitamina $D$ em 1 bilhão de indivíduos ao redor do mundo (4). 
Apesar da suposta maior exposição solar em nosso meio, concentrações insuficientes de $25(\mathrm{OH}) \mathrm{D}$ sérica são também reportadas. Saraiva e cols. (13) e de Souza Genaro e cols. (14) encontraram concentrações inadequadas em $42 \%$ de idosos na cidade de São Paulo e em $24 \%$ de mulheres com osteoporose. Em adolescentes saudáveis e adultos jovens, a prevalência foi de $60 \% \mathrm{e}$ $50 \%$, respectivamente $(15,16)$.

O marcante número de publicações que identificam uma inadequação na concentração sérica de vitamina $\mathrm{D}$ em todo o mundo tem despertado o interesse de pesquisadores, que frequentemente identificam a relação dessa vitamina não somente com a osteoporose, mas também com o desenvolvimento de doenças endocrinometabólicas $(5,6,17)$. Este artigo revisou as evidências sobre a participação da vitamina $\mathrm{D}$ nessas doenças, assim como elucidou os prováveis mecanismos de ação.

\section{DIABETES MELITO}

É bem conhecida a relação do cálcio sérico e do PTH com o desenvolvimento do diabetes melito tipo 2 (DMT2) (18); entretanto, atualmente, estudos em humanos sugerem que a $25(\mathrm{OH}) \mathrm{D}$ pode atuar como potente agente modificador do risco para o aparecimento dessa doença $(19,20)$. Estudos clínicos e epidemiológicos confirmam essa hipótese, pois demonstram que indivíduos com redução na concentração de $25(\mathrm{OH}) \mathrm{D}$ sérica apresentam maior risco para desenvolver DMT2 $(21,23)$.

O desenvolvimento de DMT2 envolve alterações na função das células- $\beta$ do pâncreas e resistência periférica à ação da insulina. A $25(\mathrm{OH}) \mathrm{D}$ pode atuar nesses mecanismos em virtude da presença (VDR) nas células- $\beta$ e de proteínas ligadoras de cálcio dependente de vitamina D (DBP) no tecido pancreático (24).

A vitamina $\mathrm{D}$ pode afetar a resposta insulínica ao estímulo da glicose direta ou indiretamente (25). O efeito direto parece ser mediado pela ligação da $1,25(\mathrm{OH})_{2} \mathrm{D}_{3}$ ao VDR da célula- $\beta$. Alternativamente, a ativação da vitamina $\mathrm{D}$ pode ocorrer dentro das células- $\beta$ pela enzima $1 \alpha$-hidroxilase, expressa nessas células (26).

$\mathrm{O}$ efeito indireto é mediado pelo fluxo de cálcio intra e extracelular nas células- $\beta$. Zemel (17) demonstrou que o aumento na $1,25(\mathrm{OH})_{2} \mathrm{D}_{3}$ e no PTH induz maior influxo de cálcio para o interior das células. Como a secreção de insulina é um processo cálcio-dependente mediado pela $1,25(\mathrm{OH})_{2} \mathrm{D}_{3}$ e pelo PTH, o aumento nas concentrações destes, devido à insuficiência de $25(\mathrm{OH}) \mathrm{D}$, pode reduzir a capacidade secretora dessas células $(25,27)$. Adicionalmente, a deficiência de $25(\mathrm{OH}) \mathrm{D}$ parece dificultar a capacidade das células- $\beta$ na conversão da pró-insulina à insulina $(28,29)$.

Com relação à ação da $25(\mathrm{OH}) \mathrm{D}$ na resistência à insulina, os efeitos podem também ser diretos (via estímulo da vitamina D para expressão do receptor da insulina, aumentando, assim, a resposta insulínica ao estímulo da glicose) ou indiretos (via concentração de cálcio intracelular) (30). O cálcio intracelular é essencial para mediar a resposta insulínica nos tecidos muscular e adiposo; desse modo, alterações na concentração de cálcio nesses tecidos podem contribuir para elevar a resistência periférica à ação da insulina, via redução da transdução de sinal e redução na atividade do transportador de glicose 4 (GLUT-4) (23). De fato, alguns autores $(22,31)$, mas não todos $(32)$, encontraram associação inversa entre o status da vitamina $\mathrm{D}$ e/ou do cálcio à resistência à insulina.

\section{Evidências em estudos in vivo e em humanos}

Ismail e Namala (27) demonstraram em ratos Wistar que uma dieta deficiente em vitamina $\mathrm{D}$ prejudica a tolerância à glicose, causando a alteração da sensibilidade à insulina. Em camundongos com VDR inativo nas ilhotas pancreáticas, observou-se diminuição na tolerância à glicose, quando eles eram submetidos à sobrecarga oral ou subcutânea de glicose. Adicionalmente, os autores observaram diminuição no mRNA da insulina (25). Por outro lado, Mathieu e cols. (33) não encontraram o mesmo efeito. Esses resultados controversos podem ser atribuídos às diferentes linhagens genéticas usadas para gerar esses animais sem VDR.

Em humanos, há também evidências da associação inversa entre resistência à insulina e $25(\mathrm{OH}) \mathrm{D}$. Alguns autores $(34,35)$ observaram reduzidas concentrações séricas de $25(\mathrm{OH}) \mathrm{D}$ em indivíduos com intolerância à glicose e/ou com diabetes. Chiu e cols. (22) avaliaram a associação entre $25(\mathrm{OH}) \mathrm{D}$ sérica e sensibilidade à insulina e função das células- $\beta$, por meio da técnica do clamp de insulina em indivíduos saudáveis, além de demonstrar correlação positiva entre $25(\mathrm{OH}) \mathrm{D}$ e o índice de sensibilidade à insulina. Liu e cols. (36), analisando a existência de relação entre $25(\mathrm{OH}) \mathrm{D}$ e resistência à insulina representada pelo modelo de avaliação da homeostase da sensibilidade à insulina (HOMA-IR) e o índice de sensibilidade à insulina ( ISI $_{0-120}$ ) em 808 participantes não diabéticos do Framingham Offspring Study, encontraram que as concentrações séricas de $25(\mathrm{OH})$ $\mathrm{D}$ foram inversamente associadas às concentrações de 
glicemia de jejum, insulina de jejum e HOMA-IR. A concentração de $25(\mathrm{OH}) \mathrm{D}$ foi positivamente associada com ISI $_{0-120}$, adiponectina e colesterol de alta densidade (HDL) e inversamente associada a triacilgliceróis.

Corroborando esses dados, estudo de coorte realizado na Finlândia comprovou associação inversa entre $25(\mathrm{OH}) \mathrm{D}$ e o risco de desenvolvimento de DMT2. Matilla e cols. (37), avaliando 4.423 indivíduos de ambos os sexos e idade média entre 40 e 69 anos, com o objetivo de determinar a incidência de DMT2, encontraram risco relativo para desenvolver diabetes melito de 0,7 (IC 95\% 0,42-1,16; P trend = 0,07), ajustado para índice de massa corpórea (IMC), exercício nas horas de lazer, fumo e nível educacional, quando comparados o maior e o menor quintil de concentrações de 25(OH)D.

\section{Evidências de estudos de intervenção}

Resultados de estudos referentes à suplementação com $25(\mathrm{OH}) \mathrm{D}$ sugerem melhora na secreção de insulina em indivíduos com DMT2. Borissova e cols. (38) observaram que o aumento na $25(\mathrm{OH}) \mathrm{D}$ sérica de 10 para $30 \mathrm{ng} / \mathrm{mL}$ pode melhorar a sensibilidade à insulina em $60 \%$. Outros pesquisadores confirmam que a suplementação de $25(\mathrm{OH}) \mathrm{D}$ em humanos pode aumentar a secreção de insulina estimulada em resposta à carga de glicose oral em pacientes com DMT2 (sem glicemia de jejum alterada) em adultos saudáveis não diabéticos e em indivíduos com deficiência de vitamina $\mathrm{D}$ sem DMT2 estabelecida $(22,32)$.

Pittas e cols. (39), analisando a população do Nurses' Health Study, com 84 mil mulheres sem história de DMT2 no início do estudo, encontraram incidência de 4.843 casos de diabetes decorridos 20 anos nessa população. O risco relativo para DMT2 foi de 0,87 (IC 95\% 0,75-1,00; P trend = 0,04), quando comparada a maior (800UI) com a menor (400UI) concentração de suplementação de $25(\mathrm{OH}) \mathrm{D}$.

De fato, a suplementação de vitamina $\mathrm{D}$ em concentração de 400UI também não mostrou efeito positivo na prevenção de DMT2 no Women's Heatlth Initiative, estudo prospectivo placebo-controlado com 33.951 mulheres, durante sete anos (40).

Em 2007, uma metanálise de estudos clínicos e observacionais sobre o papel da $25(\mathrm{OH}) \mathrm{D}$ e do cálcio em relação ao DMT2 foi realizada. Os estudos observacionais demonstram que o baixo status de $25(\mathrm{OH}) \mathrm{D}$, cálcio e derivados do leite está relacionado à maior prevalência de DMT2 e síndrome metabólica. Nos indivíduos com maiores concentrações de $25(\mathrm{OH}) \mathrm{D}$, a razão de chance para desenvolver DMT2 foi de 0,36 (IC95\% = 0,16$0,80)$, quando estes foram comparados aos indivíduos com menores concentrações. $\mathrm{O}$ mesmo fator protetor esteve presente para a incidência de DMT2, em que a razão de chance foi $0,82(\mathrm{IC} 95 \%=0,72-0,93)$. Além disso, os estudos de intervenção sugerem que a suplementação de $25(\mathrm{OH}) \mathrm{D}$ e/ou cálcio parece ser importante para a prevenção de DMT2, particularmente em indivíduos com intolerância à glicose $(23)$.

\section{Comparação dos efeitos da vitamina D com mudanças no estilo de vida e medicamentos no controle glicêmico}

Utilizando dados de estudo de suplementação com 700UI de vitamina $\mathrm{D}_{3}$ e $500 \mathrm{mg}$ de cálcio, durante três anos, em mulheres com mais de 65 anos, Pittas e cols. (41) demonstraram redução na glicemia de jejum semelhante à observada no estudo de prevenção de diabetes do Diabetes Prevention Program (DPP) por meio de mudanças do estilo de vida e também pelo uso da metformina (42). Esses dados estão ilustrados na figura 1.

Apesar da semelhança na concentração da glicemia de jejum, ainda são necessários estudos futuros que comparem o efeito da adequação do status da vitamina D somado às mudanças no estilo de vida na prevenção do DMT2.

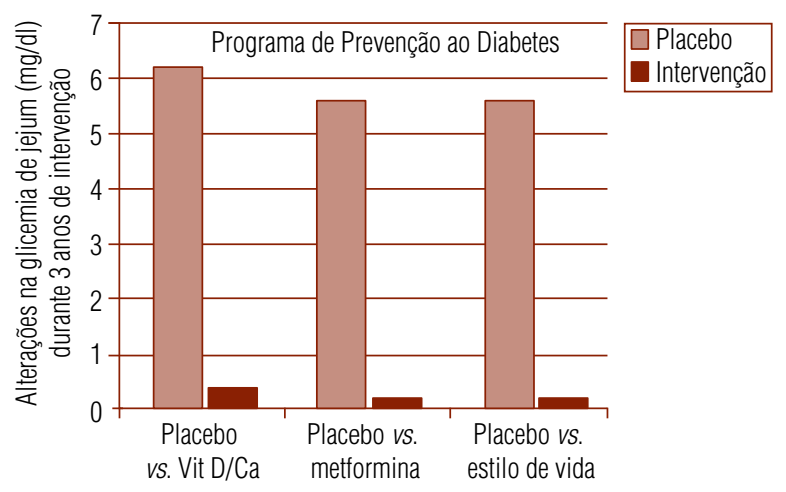

Figura 1. Vitamina D - Cálcio vs. intervenção para prevenção do diabetes tipo 2. (Adaptado de Pittas e cols. (23).

Em resumo, os resultados dos estudos sobre os efeitos da vitamina $\mathrm{D}$ no diabetes melito evidenciam que a deficiência de $25(\mathrm{OH}) \mathrm{D}$ altera a síntese e a secreção de insulina, tanto em modelos animais como em humanos. Além disso, a deficiência dessa vitamina parece acarretar a intolerância à glicose e a secreção de insulina prejudicada e contribuir para o maior risco de DMT2. Entretanto, o diabetes melito é uma doença multifatorial, 
cuja prevenção envolve alterações significantes no estilo de vida, como adequação na alimentação e na prática de atividade física. As recentes evidências da participação da vitamina $\mathrm{D}$ nos processos de secreção e ação da insulina e no controle glicêmico sugerem que a vitamina D deve ser mais um fator considerado tanto na prevenção como no tratamento do diabetes melito.

\section{OBESIDADE}

Reduzidas concentrações de $25(\mathrm{OH}) \mathrm{D}$ são frequentemente observadas em indivíduos obesos (43-47). Especula-se que a insuficiência de vitamina $\mathrm{D}$ não seja apenas consequência da menor exposição solar em obesos, mas também um dos fatores que desencadeia o acúmulo de gordura corporal.

Evidências sugerem que uma das causas da deficiência de $25(\mathrm{OH}) \mathrm{D}$ em indivíduos obesos e com DMT2 possa estar ligada ao depósito de vitamina D nos adipócitos, diminuindo a sua biodisponibilidade e acionando o hipotálamo para desenvolver uma cascata de reações que resulta no aumento da sensação de fome e na diminuição do gasto energético (48). Tal situação também gera aumento nos níveis de PTH, consequente diminuição da sensibilidade à insulina e aumento desproporcional na concentração de cálcio intracelular $(44,49-52)$.

\section{Evidências em estudos epidemiológicos}

Correlação negativa entre gordura corporal total com concentração sérica de $25(\mathrm{OH}) \mathrm{D}$ foi demonstrada em estudos de base populacional. Tal correlação permaneceu significante, mesmo após o ajuste para idade, estação do ano, ingestão de vitamina D e raça $(43,44)$.

Snidjer e cols. (44), no Longitudinal Aging Study Amsterdam (LASA), observaram que a soma das dobras cutâneas e o percentual de gordura corporal estiveram fortemente associados à baixa concentração de $25(\mathrm{OH})$ $\mathrm{D}$ e à maior concentração de $\mathrm{PTH}$.

A hipótese de que indivíduos obesos apresentam maiores concentrações de PTH foi confirmada por Bell e cols. (45). Maior secreção de PTH é sinal para hidroxilação da forma ativa da vitamina $\mathrm{D}$. Os autores sugerem que, devido à elevação na $1,25(\mathrm{OH})_{2} \mathrm{D}_{3}$, ocorra feedback negativo à síntese hepática de $25(\mathrm{OH}) \mathrm{D}$.

Posteriormente, estudo avaliando as concentrações de micronutrientes em homens e mulheres que participaram do National Health and Nutrition Examination Survey III (NHANES III) demonstrou que somente nas mulheres pré e pós-menopausadas $(\mathrm{n}=8.137)$ houve associação negativa da vitamina $\mathrm{D}$ com o IMC (46). Entretanto, associação da relação entre obesidade e PTH não foi demonstrada.

\section{Evidências in vivo e in vittro}

Foi demonstrado que, em culturas de adipócitos, o PTH $(47)$ e a $1,25(\mathrm{OH})_{2} \mathrm{D}_{3}$ podem elevar a concentração de cálcio intracelular, fato que impede a lipólise induzida por catecolaminas e promove a expressão do ácido graxo sintase, contribuindo para o acúmulo de gordura (48).

Recentes estudos feitos com modelos de culturas de pré-adipócitos de camundongos levantaram a hipótese de que o papel da 25(OH)D na inibição da adipogênese é mediado em nível molecular por meio da inibição do VDR e da expressão do regulador-mestre da adipogênese (PPAR $\gamma$ ), reduzindo, consequentemente, sua atividade (49). No entanto, estudos realizados há mais de 20 anos indicaram que culturas de pré-adipócitos tratados com $1,25(\mathrm{OH})_{2} \mathrm{D}_{3}$ tiveram sua diferenciação para adipócito afetada. Esse estudo mostrou que concentrações nanomolares de $1,25(\mathrm{OH})_{2} \mathrm{D}_{3}$ podem inibir a adipogênese e reduzir o acúmulo de triacilglicerol em até $50 \%$, em comparação com as células-controle diferenciadas (24). Essas descobertas são condizentes com a ideia de que qualquer influência da $1,25(\mathrm{OH})_{2} \mathrm{D}_{3}$ na adipogênese poderia ser exercida antes da transição de pré-adipócito para o adipócito, quando existe maior disponibilidade de VDR. Essa importante observação sugere também que o papel principal da $1,25(\mathrm{OH})_{2} \mathrm{D}_{3}$ na adipogênese parece ser a supressão de um evento molecular-chave primário ou anterior no processo de diferenciação do pré-adipócito (49).

De fato, Blumberg e cols. (50), utilizando uma linhagem de células de pré-adipócitos 3T3-L15B2, demonstraram que o tratamento com a $1,25(\mathrm{OH})_{2} \mathrm{D}_{3}$ inibiu parcialmente a formação endógena de ligantes de PPAR . Assim, com a redução da expressão do PPAR $\gamma$ e consequente diminuição de sua atividade na presença de $1,25(\mathrm{OH})_{2} \mathrm{D}_{3}$, ocorre inibição da adipogênese. Contudo, pesquisas adicionais são necessárias para melhor esclarecer o decréscimo de PPAR $\gamma$ na presença de $1,25(\mathrm{OH})_{2} \mathrm{D}_{3} \mathrm{e}$ investigar a extensão da interação entre VDR e PPAR $\gamma$ em pré-adipócitos in vivo (49).

\section{Evidências em estudo de suplementação}

Caan e cols. (51), analisando dados de 36.282 mulheres na pós-menopausa com idade entre 50 e 79 anos 
participantes do Women's Health Initiative (WHI), encontraram menor ganho de peso durante o uso de suplementação ( $1.000 \mathrm{mg}$ de cálcio e 400UI de colecalciferol) quando comparadas ao grupo controle.

Estudo duplo-cego placebo-controlado, realizado com 445 indivíduos de 21 a 70 anos e IMC entre 28 e $47 \mathrm{~kg} / \mathrm{m}^{2}$, utilizou suplementação de colecalciferol em três grupos:

l) 20.000UI duas vezes por semana;

2) 20.000UI uma vez por semana + placebo uma vez por semana;

3) placebo duas vezes por semana.

Todos os participantes receberam $500 \mathrm{mg}$ de cálcio. Houve aumento significante na concentração de $25(\mathrm{OH}) \mathrm{D}$ e redução no $\mathrm{PTH}$, mas não houve mudanças significantes em peso, relação cintura-quadril e percentual de gordura corporal intragrupo e intergrupo, mesmo quando avaliados a partir das concentrações séricas basais de $25(\mathrm{OH}) \mathrm{D}(52)$. Entretanto, estudos que avaliem os efeitos da suplementação da vitamina $\mathrm{D}$ na prevenção da obesidade ainda são necessários.

\section{HIPERTENSÃO}

Dados do Intersalt Study, importante trabalho sobre fatores de risco e controle de hipertensão com mais de 10.000 indivíduos de diversos países, mostram pressão arterial sistólica e diastólica positivamente associada à distância do equador, elucidando que a exposição solar e, supostamente, a menor concentração de $25(\mathrm{OH}) \mathrm{D}$ estariam relacionadas à pressão arterial (53).

Adicionalmente, em estudo realizado por Krause e cols. (54) com pacientes hipertensos submetidos à radiação ultravioleta três vezes por semana, durante três meses, foi demonstrado aumento de $180 \%$ nos níveis séricos de $25(\mathrm{OH}) \mathrm{D}$ e redução de $6 \mathrm{mmHg}$ na pressão arterial sistólica e diastólica.

A hipertensão ocorre principalmente pela ativação inadequada do sistema renina-angiotensina. São vários os estudos que apontam níveis séricos de $1,25\left(\mathrm{OH}_{2}\right) \mathrm{D}_{3}$ inversamente associados à pressão arterial ou à atividade da renina plasmática em normotensos e hipertensos (55-57). A ação da $1,25\left(\mathrm{OH}_{2}\right) \mathrm{D}_{3}$ influenciando a expressão gênica ocorre por meio do receptor de vitamina $\mathrm{D}$ (VDR) presente em vários tecidos/células, como no aparelho justaglomerular (58). Em 2008, Kong e cols. (59) demonstraram que a supressão da expressão de renina pela $1,25\left(\mathrm{OH}_{2}\right) \mathrm{D}_{3}$ in vivo é independente do $\mathrm{PTH}$ e do cálcio.
Estudos experimentais demonstraram que a $1,25\left(\mathrm{OH}_{2}\right) \mathrm{D}_{3}$ inibe a expressão da renina no aparelho justaglomerular (60) e bloqueia a proliferação de célula vascular muscular lisa (VSMC) (61). Assim, a relação vitamina $\mathrm{D} /$ hipertensão pode ocorrer via sistema renina-angiotensina e função vascular. Além disso, a 1- $\alpha$ hydroxilase, enzima de conversão da $25(\mathrm{OH}) \mathrm{D}$ em $1,25\left(\mathrm{OH}_{2}\right) \mathrm{D}_{3}$, tem expressão em diversos tecidos, como células endoteliais, VSMC, além das células renais $(62,63)$, sugerindo um efeito parácrino da $25(\mathrm{OH}) \mathrm{D}$ independente dos níveis circulantes de $1,25\left(\mathrm{OH}_{2}\right) \mathrm{D}_{3}$.

\section{Evidências em estudos epidemiológicos}

Analisando a população com mais de 20 anos que participou do NHANES III, Scragg e cols. (64) encontraram pressão arterial sistólica e diastólica $(3,0$ e $1,6 \mathrm{mmHg}$, respectivamente) menor no maior quintil $(25(\mathrm{OH}) \mathrm{D} \geq 85,7 \mathrm{nmol} / \mathrm{L})$, em comparação ao menor quintil de vitamina $\mathrm{D}(25(\mathrm{OH}) \mathrm{D} \leq 40 \mathrm{nmol} / \mathrm{L})$. Adicionalmente, Martins e cols. (65) encontraram em adultos americanos prevalência de hipertensão $30 \%$ maior no menor quartil, quando comparada ao maior quartil de vitamina $D$.

Recentemente, a associação entre os níveis séricos de $25(\mathrm{OH}) \mathrm{D}$ e o risco de doença coronariana nos indivíduos que participaram do Health Professionals Followup Study (HPFS) foi avaliada. Homens com deficiência de vitamina $\mathrm{D}(\leq 15 \mathrm{ng} / \mathrm{mL}$ ou $37 \mathrm{nmol} / \mathrm{L})$ apresentam risco significantemente maior de desenvolver infarto do miocárdio quando comparados àqueles com níveis suficientes de vitamina $\mathrm{D}(\geq 30 \mathrm{ng} / \mathrm{mL}$ ou $75 \mathrm{nmol} / \mathrm{L})$ $(\mathrm{RR}=2,09$; IC95\% = 1,24-3,54) (66). Resultados semelhantes foram observados por Kendrick e cols. (67) ao avaliarem a população acima de 18 anos do NHANES III, na qual indivíduos com deficiência de vitamina D $(25(\mathrm{OH}) \mathrm{D}<20 \mathrm{ng} / \mathrm{mL})$ apresentaram maior risco de doença cardiovascular $(\mathrm{OR}=1,2 ; \mathrm{IC} 95 \%=1,01-1,36)$ após os ajustes por potenciais fatores de confusão.

Utilizando ainda os dados do HPFS e do Nurses' Health Study, a relação negativa entre níveis séricos de vitamina $\mathrm{D}$ e hipertensão foi também demonstrada (68). Aos quatro anos de seguimento, o risco relativo, para homens com reduzidos níveis de $25(\mathrm{OH}) \mathrm{D}$ sérica desenvolverem hipertensão, foi de 6,13 (IC95\% $=1,00-37,80)$, enquanto, nas mulheres, foi de 2,67 (IC95\% = 1,05-6,97). Após oito anos de seguimento, nos homens, o risco relativo foi de 3,53 (IC95\% $=1,02$ 12,3) e, nas mulheres, 1,7 (IC95\% = 0,92-3,16). 
Em outra análise, esta com 1.484 mulheres participantes do Nurses' Health Study que foram divididas em 742 casos que desenvolveram hipertensão e em 742 controles que não desenvolveram hipertensão, foi observado que a concentração de $25(\mathrm{OH}) \mathrm{D}$ era menor nos casos $(25,6 \mathrm{ng} / \mathrm{mL})$ do que nos controles $(27,3$ $\mathrm{ng} / \mathrm{mL} ; \mathrm{p}=0,00 \mathrm{l})$. As mulheres que se encontravam no menor quartil de vitamina $\mathrm{D}$ apresentaram maior risco de desenvolver hipertensão do que as mulheres no maior quartil (razão de chance ajustada de 1,66; IC95\% = 1,11-2,48; p = 0,01). O mesmo foi observado quando comparadas mulheres com níveis suficientes e deficientes de vitamina $\mathrm{D}$ (razão de chance ajustada de $1,47 ;$ IC95\% $=1,10-1,97)(69)$.

\section{Evidências in vivo}

Zhou e cols. (70) demonstraram regulação do sistema renina-angiotensina por meio da suplementação de $1,25\left(\mathrm{OH}_{2}\right) \mathrm{D}_{3}$ em ratos isentos da enzima l- $\alpha$ hydroxilase. Entretanto, Thierry-Palmer e cols. (71) aumentaram a oferta de vitamina $\mathrm{D}$ pela dieta em ratos sal-sensíveis com alimentação salgada e, apesar do aumento dos níveis séricos de $25(\mathrm{OH}) \mathrm{D}$, a hipertensão não foi atenuada.

\section{Evidências em estudos de suplementação}

Em estudo duplo-cego placebo-controlado, foi observada a redução na pressão arterial de 39 indivíduos hipertensos com a suplementação de vitamina D (72). Essa redução também foi ressaltada em mulheres idosas suplementadas com cálcio e vitamina D (73).

Em outro ensaio clínico, foi observado que administração de $1,25\left(\mathrm{OH}_{2}\right) \mathrm{D}_{3}$ reduziu a pressão arterial, além da atividade da renina plasmática e dos níveis de angiotensina II (74).

No entanto, ainda é preciso avaliar os efeitos da suplementação de $25(\mathrm{OH}) \mathrm{D}$ em estudos de base populacional e também em subgrupos específicos na prevenção da hipertensão arterial, além de analisar o status da vitamina $\mathrm{D}$ necessário em diferentes populações para garantir o benefício máximo desta na pressão arterial.

\section{CONSIDERAÇÕES FINAIS}

Evidências recentes demonstram que a insuficiência da vitamina $\mathrm{D}$ pode estar relacionada ao diabetes melito, à obesidade e à hipertensão. Entretanto, estudos prospectivos e de intervenção em humanos que comprovem a efetividade da adequação do status da vitamina $\mathrm{D}$, tanto na prevenção como no tratamento dessas doenças, ainda são escassos. Mais ainda, a compreensão dos mecanismos exatos pelos quais a $25(\mathrm{OH}) \mathrm{D}$ ou a forma ativa $1,25(\mathrm{OH})_{2} \mathrm{D}_{3}$ promovem melhor funcionamento das células- $\beta$, do sistema renina-angiotensina e da regulação da quantidade de gordura corporal são também incompletos.

Contudo, considerando que a insuficiência de vitamina D é comumente observada e que há importantes lacunas no conhecimento sobre a ação dessa vitamina em relação à prevenção e ao tratamento de doenças endocrinometabólicas, a investigação da adequação do status da vitamina D nessas situações deve ser objeto de futuras pesquisas.

Declaração: os autores declaram não haver conflitos de interesse científico neste estudo.

\section{REFERÊNCIAS}

1. Bouillon R, Carmeliet G, Verlinden L, van Etten E, Verstuyf A, Luderer $\mathrm{HF}$, et al. Vitamin $\mathrm{D}$ and human health: lessons from vitamin D receptor null mice. Endocr Rev. 2008;29(6):726-76.

2. Heaney RP, Armas LAG, Shary JR, Bell NH, Binkley N, Hollis BW. 25hydroxilation of vitamin D3: relation to circulating vitamin D3 under various input conditions. Am J Clin Nut. 2008; 87(6):1738-42.

3. Kimball S, Fuleihan Gel-H, Vieth R. Vitamin D: a growing perspective. Crit Rev Clin Lab Sci. 2008;45(4):339-414.

4. James WP. 22nd Marabou Symposium: the changing faces of vitamin D. Nut Rev. 2008;66(5):286-90.

5. Peterlick M, Cross HS. Vitamin D and calcium deficits predispose for multiple chronic diseases. Eur J Clin Invest. 2005;35(5):290-304.

6. Prentice A, Goldberg G, Schoenmakers I. Vitamin D across the lifecycle: physiology and biomarkers. Am J Clin Nut. 2008;88(2):500S-506S.

7. Mosekilde L. Vitamin D and the elderly. Clin Endocrinol. 2005; 62(3):265-81.

8. Hollis BW. Circulating 25-hydroxyvitamin D levels indicative of vitamin $D$ sufficiency: implications for establishing a new effective dietary intake recommendation for vitamin D. J Nutr. 2005;135(2):317-22.

9. Chapuy MC, Preziosi P, Maamer M, Arnaud S, Galan P, Hercberg $S$, et al. Prevalence of vitamin D insufficiency in an adult normal population. Osteoporos Int. 1997;7(5):439-43.

10. Tangpricha V, Pearce EN, Chen TC, Holick MF. Vitamin D insufficiency among free-living healthy young adults. Am J Med. 2002;112(8):659-62.

11. Vieth R, Ladak $Y$, Walsh PG. Age-related changes in the 25-hydroxyvitamin $\mathrm{D}$ versus parathyroid hormone relationship suggest a different reason why older adults require more vitamin D. J Clin Endocrinol Metab. 2003;88(1):185-91.

12. Grant WB, Holick MF. Benefits and requirements of vitamin $D$ for optimal health: a review. Altern Med Rev. 2005;10(2):94-111.

13. Saraiva GL, Cendoroglo MS, Ramos LR, Araújo LMQ, Vieira JGH, Kunii I, et al. Influence of ultraviolet radiation on the production of 25 hidroxyvitamin $D$ in the elderly population in the city of São Paulo (23 o 34'S), Brazil. Osteoporos Int. 2005;16(12):1649-54.

14. de Souza Genaro P, de Paiva Pereira GA, de Medeiros Pinheiro M, Szejnfeld VL, Araújo Martini L. Relationship between nutrient 
intake and vitamin D status in osteoporotic women. Int J Vitam Nutr Res. 2007;77(6):376-81.

15. Peters BSE, dos Santos LC, Fisberg M, Wood RJ, Martini LA. Prevalence of vitamin $D$ insufficiency in Brazilian adolescents. Ann Nutr Metab. 2009;54(1):15-21.

16. Maeda SS, Kuni IS, Hayashi L, Lazaretti-Castro M. The effects of sun exposure on 25-hydroxyvitamin $D$ concentrations in young healthy subjects living in the city of São Paulo, Brazil. Braz J Med Biol Res. 2007;40(12):1653-9.

17. Zemel MB. Mechanisms of dairy modulation of adiposity. J Nutr. 2003;133:252S-256S.

18. Levy J. Abnormal cell calcium homeostasis in type 2 diabetes mellitus: a new look on old disease. Endocrine. 1999;10(1):1-6.

19. Gregori S, Giarratana N, Smiroldo S, Uskokovic M, Adorini L. A 1alpha,25-dihydroxyvitamin $D(3)$ analog enhances regulatory $T$ cells and arrests autoimmune diabetes in NOD mice. Diabetes. 2002;51(5):1367-74.

20. Zipitis CS, Akobeng AK. Vitamin D supplementation in early childhood and risk of type 1 diabetes: a systematic review and metaanalysis. Arch Dis Child. 2008;93(6):512-7.

21. Hyppönen $E$, Lärä $E$, Reunanen $A$, Järvelin $M-R$, Virtanen SM. Intake of vitamin $D$ and risk of type 1 diabetes: a birth-cohort study. Lancet. 2001;358(9292):1500-3.

22. Chiu KC, Chu A, Go VL, Saad MF. Hypovitaminosis D is associated with insulin resistance and beta cell dysfunction. Am J Clin Nutr. 2004;79(5):820-5.

23. Pittas AG, Lau J, Hu FB, Dawson-Hughes B. The role of vitamin D and calcium in type 2 diabetes. A systematic review and metaanalysis. J Clin Endocrinol Metab. 2007;92(6):2017-29.

24. Ishida $\mathrm{H}$, Norman AW. Demonstration of a high affinity receptor for 1,25-dihydroxyvitamin D3 in rat pancreas. Moll Cell Endocrinol. 1988;60(2-3):109-17.

25. Zeitz U, Weber K, Soegiarto DW, Wolf E, Balling R, Erben RG. Impaired insulin secretory capacity in mice lacking a functional vitamin D receptor. FASEB J. 2003;17(3):509-11.

26. Bland R, Markovic D, Hills CE, Hughes SV, Chan SL, Squires PE, et al. Expression of 25-hydroxivitamin D3-alpha-hydroxylase in pancreatic islets. J Steroid Biochem Mol Biol. 2004;89-90(1-5):121-5.

27. Ismail A, Namala R. Impaired glucose tolerance in vitamin D deficiency can be corrected by calcium. J Nutr Biochem. 2000;11(3):170-5.

28. Bourlon PM, Billaudel B, Faure-Dussert A. Influence of vitamin D3 deficiency and 1,25 dihydroxyvitamin D3 on de novo insulin biosynthesis in the islets of the rat endocrine pancreas. J Endocrinol. 1999;160(1):87-95.

29. Ayesha I, Bala TS, Reddy CV, Raghuramulu N. Vitamin D deficiency reduces insulin secretion and turnover in rats. Diabetes Nutr Metab. 2001;14(2):78-84.

30. Maestro B, Campión J, Dávila N, Calle C. Stimulation by 1,25dihydroxyvitamin D3 of insulin receptor expression and insulin responsiveness for glucose transport in U-937 human promonocytic cells. Endocr J. 2000;47(4):383-91.

31. Scragg R, Sowers M, Bell C; Third National Health and Nutrition Examination Survey. Serum 25-hydroxyvitamin D, diabetes, and ethnicity in the Third National Health and Nutrition Examination Survey. Diabetes Care. 2004;27(12):2813-8.

32. Orwoll $E$, Riddle $M$, Prince $M$. Effects of vitamin $D$ on insulin and glucagon secretion in non-insulin-dependent diabetes mellitus. Am J Clin Nutr. 1994;59(5):1083-7.

33. Mathieu C, Van Etten E, Gysemans C, Decallonne B, Kato S, Laureys $\mathrm{J}$, et al. In vitro and in vivo analysis of the immune system of vitamin $\mathrm{D}$ receptor knockout mice. J Bone Miner Res. 2001;16(11):2057-65.
34. Boucher BJ, Mannan N, Noonan K, Hales CN, Evans SJ. Glucose intolerance and impairment of insulin secretion in relation to vitamin D deficiency in east London Asians. Diabetologia. 1995; 38(10):1239-45.

35. Morris KL, Zemel MB. 1,25-dihydroxyvitamin D3 modulation of adipocyte glucocorticoid function. Obes Res. 2005;13(4):670-9.

36. Liu E, Meigs JB, Pittas AG, McKeown NM, Economos CD, Booth $\mathrm{SL}$, et al. Plasma 25 -hydroxyvitamin $\mathrm{D}$ is associated with markers of the insulin resistant phenotype in nondiabetic adults. J Nutr. 2009;139(2):329-34.

37. Mattila C, Knekt P, Männistö S, Rissanen H, Laaksonen MA, Montonen J, et al. Serum 25-hydroxyvitamin D concentration and subsequent risk of type 2 diabetes. Diabetes Care. 2007;30(10):2569-70.

38. Borissova AM, Tankova T, Kirilov G, Dakovska L, Kovacheva R. The effect of vitamin D3 on insulin secretion and peripheral insulin sensitivity in type 2 diabetic patients. Int J Clin Pract. 2003;57(4):258-61.

39. Pittas AG, Dawson-Hughes B, LiT, Van Dam RM, Willett WC, Manson $\mathrm{JE}$, et al. Vitamin $\mathrm{D}$ and calcium intake in relation to type 2 diabetes in women. Diabetes Care. 2006; 29(3):650-6.

40. de Boer IH, Tinker LF, Connelly S, Curb JD, Howard BV, Kestenbaum B, Larson JC, Manson JE, Margolis KL, Siscovick DS, Weiss NS; Women's Health Initiative Investigators. Calcium plus vitamin D supplementation and the risk of incident diabetes in the Women's Health Initiative. Diabetes Care. 2008;31(4):701-7.

41. 41. Pittas AG, Harris SS, Stark PC, Dawson-Hughes B. The effects of calcium and vitamin $D$ supplementation on blood glucose and markers of inflammation in nondiabetic adults. Diabetes Care. 2007;30(4):980-6.

42. Knowler WC, Barrett-Connor E, Fowler SE, Hamman RF, Lachin JM, Walker EA, Nathan DM; Diabetes Prevention Program Research Group. Reduction in the incidence of type 2 diabetes with lifestyle intervention or metformin. 2002;346(6):393-403.

43. Arunabh S, Pollack S, Yeh J, Aloia JF. Body fat content and 25hydroxivitamin $\mathrm{D}$ levels in healthy women. $\mathrm{J}$ Clin Endocrinol Metab. 2003;88(1):157-61.

44. Snijder MB, van Dam RM, Visser M, Deeg DJ, Dekker JM, Bouter $\mathrm{LM}$, et al. Adiposity in relation to vitamin $\mathrm{D}$ status and parathyroid hormone levels: a population-based study in older men and women. J Clin Endocrinol Metab. 2005;90(7):4119-23.

45. Bell NH, Epstein S, Greene A, Shary J, Oexmann MJ, Shaw S. Evidence for alteration of the vitamin D-endocrine system in obese subjects. J Clin Invest. 1985;76(1):370-3.

46. Kimmons JE, Blanck HM, Tohill BC. Associations between body mass index and the prevalence of low micronutrient levels among US adults. Med Gen Med. 2006;8(4):59.

47. Ni Z, Smogorjewski M, Massry SG. Effects of parathyroid hormone on cytosolic calcium of rat adipocytes. Endocrinology. 1994; 135(5):1837-44.

48. Su X, Zemel MB. 1alpha, 25-dihydroxyvitamin D and corticosteroid regulate adipocyte nuclear vitamin $\mathrm{D}$ receptor. Int $\mathrm{J}$ Obes (Lond). 2008;32(8):1305-11.

49. Wood RJ. Vitamin D and adipogenesis: new molecular insights. Nutn Rev. 2007; 66(1):40-6.

50. Blumberg JM, Tzameli I, Astapova I, Lam FS, Flier JS, Hollenberg AN. Complex role of the vitamin D receptor and its ligand in adipogenesis in 3T3-L1 cells. J Biol Chem. 2006;281(16):11205-13.

51. Caan B, Neuhouser M, Aragaki A, Lewis CB, Jackson R, LeBoff MS, et al. Calcium plus vitamin $D$ supplementation and the risk of postmenopausal weight gain. Arch Intern Med. 2007; 14;167(9):893902.

52. Sneve M, Figenschau Y, Jorde R. Supplementation with cholecalciferol does not result in weight reduction in overweight and obese subjects. Eur J Endocrinol. 2008;159(6):675-84. 
53. INTERSALT Cooperative Research Group. Intersalt: an international study of electrolyte excretion and blood pressure. Results for 24 hour urinary sodium and potassium excretion. Intersalt Cooperative Research Group. BMJ. 1988;297(6644):319-28.

54. Krause R, Bühring M, Hopfenmüller W, Holick MF, Sharma AM. Ultraviolet B and blood pressure. Lancet. 1998;352(9129):709-10.

55. Resnick LM, Nicholson JP, Laragh JH. Calcium metabolism in essential hypertension: relationship to altered renin system activity. Fed Proc. 1986;45(12):2739-45.

56. Burgess ED, Hawkins RG, Watanabe M. Interaction of 1,25dihydroxyvitamin $\mathrm{D}$ and plasma renin activity in high renin essential hypertension. Am J Hypertens. 1990;3(12 Pt 1):903-5.

57. Imaoka M, Morimoto S, Kitano S, Fukuo F, OgiharaT. Calcium metabolism in elderly hypertensive patients: possible participation of exaggerated sodium, calcium and phosphate excretion. Clin Exp Pharmacol Physiol. 1991;18(9):631-41.

58. Li YC. Vitamin D regulation of the rennin-angiotensin system. J Cell Biochem. 2003;88(2):327-31.

59. Kong J, Qiao G, Zhang Z, Liu SQ, LiYC. Targeted vitamin D receptor expression in juxtaglomerular cells suppresses renin expression independent of parathyroid hormone and calcium. Kidney Int. 2008;74(12):1577-81.

60. Li YC, Kong J, Wei M, Chen ZF, Liu SQ, Cao LP. 1,25-dihydroxivitamin D3 is a negative endocrine regulator of renin-angiotensin system. J Clin Invest 2002;110(2):229-38.

61. Carthy EP, Yamashita W, Hsu A, Ooi BS. 1,25-dihydroxivitamin D3 and rat vascular smooth muscle cell growth. Hypertension. 1989;13(6 Pt 2):954-9

62. Merke J, Milde P, Lewieka S, Hügel U, Klaus G, Mangelsdorf DJ, et al. Identification and regulation of 1,25-dihydroxivitamin D3 receptor activity and biosynthesis of 1,25-dihydroxivitamin D3. Studies in cultured bovine aortic endothelial cells and human dermal capillaries. J Clin Invest. 1989;83(6):1903-15.

63. Zehnder D, Bland R, Walker EA, Bradwell AR, Howie AJ, Hewison $M$ et al. Expression of 25-hydroxyvitamin D3-1alpha-hydroxylase in the human kidney. J Am Soc Nephrol. 1999;10(12):2465-73.

64. Scragg R, Sowers MF, Bell C. Serum 25-hydroxyvitamin D, ethnicity, and blood pressure in the Third National Healthy and Nutrition Examination Survey. J Am Hyper. 2007;20(7):713-9.
65. Martins D, Wolf M, Pan D, Zadshir A, Tareen N, Thadhani R, et al. Prevalence of cardiovascular risk factors and the serum levels of 25-hydroxyvitamin D in the United States: data from the Third National Healthy and Nutrition Examination Survey. Arch Intern Med. 2007;167(11):1159-65.

66. Giovannucci E, Liu Y, Hollis BW, Rimm EB. 25-hydroxyvitamin $D$ and risk of myocardial infarction in men: a prospective study. Arch Intern Med. 2008;168(11):1174-80.

67. Kendrick J, Targher G, Smits G, Chonchol M. 25-Hydroxyvitamin $D$ deficiency is independently associated with cardiovascular disease in the Third National Health and Nutrition Examination Survey. Atherosclerosis. 2008:1-6.

68. Forman JP, Giovannucci E, Holmes MD, Bischoff-Ferrari HA, Tworoger SS, WilletWC, et al. Plasma 25-hydroxyvitamin D levels and risk of incident hypertension. Hypertension. 2007;49(5):1063-9.

69. Forman JP, Curhan GC, Taylor EN. Plasma 25-hydroxyvitamin D levels and risk of incident hypertension among young women. Hypertension. 2008;52(5):828-32.

70. Zhou C, Lu F, Cao K, Xu D, Goltzman D, Miao D. Calcium-independent and 1,25(OH2)D3-dependent regulation of the renin-angiotensin system in 1alpha-hydroxylase knockout mice. Kidney Int. 2008;74(2):170-9.

71. Thierry-Palmer M, Cephas S, Muttardy FF, Al-Mahmoud A. High dietary cholecalciferol increases plasma 25-hydroxycholecalciferol concentration, but does not attenuate the hypertension of Dahl salt-sensitive rats fed a high salt diet. J Steroid Biochem Mol Biol. 2008;111(1-2):7-12.

72. Lind L, Wengle $B$, Ljunghall $S$. Blood pressure is lowered by vitamin $D$ (alphacalcidol) during long-term treatment of patients with intermittent hypercalcemia. A double-blind, placebo-controlled study. Acta Med Scand. 1987;222(5):423-7.

73. Pfeifer M, Begerow B, Minne HW, Nachtigall D, Hansen C. Effects of short-term vitamin D3 and calcium supplementation on blood pressure and parathyroid hormone levels in elderly women. J Clin Endocrinol Metab. 2001;86(4):1633-7.

74. KimuraY, Kawamura M, Owada M, OshimaT, Murooka M, FujiwaraT, et al. Effectiveness of 1,25-dihydroxyvitamin D supplementation on blood pressure reduction in pseudohypoparathyroidism patient with high renin activity. Intern Med. 1999;38(1):31-5 